\title{
A Robust Variable Step-Size NLMS Algorithm Through A Combination of Robust Cost Functions
}

\author{
Insun Song, Won Il Lee, Nam Kyu Kwon, and PooGyeon Park
}

\begin{abstract}
This letter introduces a new gradient-based adaptive filtering algorithm based on a cost function that is constructed by combining two robust cost functions, which are a new tanh-type cost function and Vega's cost function. Through the approach to combine robust cost functions, the robustness of the proposed algorithm outperforms that of other adaptive algorithms. Since the proposed algorithm is derived by combining two robust cost functions, it leads to an excellent transient and steady-state behavior in high probability of impulsive measurement noise. The proposed algorithm is tested in different probability of impulsive measurement noise.
\end{abstract}

Index Terms-Adaptive filter, robust filtering, impulsive noise, normalized least-mean-square (NLMS) algorithm.

\section{INTRODUCTION}

Adaptive algorithms have played major roles in varioussignal processing applications, such as system identification, echo cancellation, channel equalization, and control in communication networks[1]-[4]. For this, the normalized least-mean-squares (NLMS) algorithm has been widely used in many applications due toits simplicity and robustness [1], [2]. However, in most realenvironments, adaptive filtering applications can be influencedby various outliers including impulsive measurement noiseand they cause the performance degradation of many adaptivefilters. To overcome this drawback, many various schemeshave been proposed [5]-[14].

When using gradient-based adaptive filtering, the nextweight estimate is determined to reduce the cost functionassociated with the aposteriori output errors by using thegradient of the cost function with respect to the current weightvector, where the step size in $(0 ; 1]$ is usually designed toenhance the rate of convergence. Therefore, the cost functionand the step size play an extremely important role in gradientbasedadaptive filtering. The most common idea to handleimpulsive measurement noise has been considered to designthe appropriate cost function, which directly determines theperformance of the algorithms.

Manuscript received August 1, 2012; revised September 3, 2012

This research was supported by the MKE(The Ministry ofKnowledge Economy), Korea, under the ITRC(InformationTechnology Research Center) support program supervised bythe NIPA(National IT Industry Promotion Agency)" (NIPA-2012-(H0301-12-1003)) and byWorld Class University program funded by the Ministry ofEducation, Science and Technology through the NationalResearch Foundation of Korea(R31-10100). This research was supported by "Development of technologies for an underwater robot based on artificial intelligence for highly sophisticated missions", which is supported by KORDI.

The authors are with the Division of Departmentof Electrical and Computer Engineering and IT Convergence Engineering,Pohang University of Science and Technology, Pohang, Gyungbuk, Republicof Korea (e-mail:weedsis@postech.ac.kr,wilee@postech.ac.kr,kwunnam@postech.ac .kr,ppg@postech.ac.kr)
Various robust cost functions using the $\mathrm{L} 1$ norm havebeen developed for impulsive-noise environments [5]-[11]. Anormalized least mean absolute deviation (NLMAD) algorithm[5], a dual sign algorithm [6], variable step-size sign algorithms[7], [8], and an affine projection sign algorithm [9] arebased on the $L 1$-norm minimization, thus the algorithms usethe sign of the output error to update next weight estimate.A robust mixed-norm adaptive algorithm is based on a robustcost function using the convex combination of the $L 1$ and $L 2$ norms [10]. A robust variable-step-size (VSS) NLMSalgorithm uses a robust cost function which switches betweenthe $L 1$ and $L 2$ norms [11]. It is associated with how thealgorithm constrains the energy of the filter update at eachiteration. Various adaptive algorithms use other robust costfunctions for robustness against impulsive measurement noise[12]-[14]. When the magnitude of the output error is largerthan a threshold, the Huber mixed-norm M-estimate costfunction uses the $L 1$ norm minimization [12], on the otherhand, the Hampel three-part redescending M-estimate costfunction sets the error signal as a constant value [13], [14].

In this paper, we propose anew gradient-based adaptive filtering algorithm based on a cost function that is constructed by combining two robust cost functions. One is a new cost function which is developed by investigating and modifying the tanh cost function and the latter is a robust cost function which Vega proposed in [11]. Through our approach to combine cost functions, the robustness of the proposed algorithm outperforms that of other adaptive algorithms. Since the proposed algorithm is derived by combining two robust cost functions, itleads to an excellent transient and steady-state behaviorin high probability of impulsive measurement noise. Using simulations, the proposed algorithm is checked the robustness against impulsive measurement noise and we compared it with various robust adaptive algorithms in impulsive-noiseenvironment.

\section{New Robust Cost Functions}

\section{A. Tanh Cost Function}

Insystem identification, the desired signal $d_{i}$ is represented as

$$
d_{i}=\mathbf{u}_{i}^{T} \mathbf{w}+\bar{v}_{i}
$$

where, $\mathbf{w} \in \Re^{n}$ is a coefficient vector of the unknown system, the input vector $\mathbf{u}_{i}$ denotes $\left[x_{i}, \ldots, x_{i-n+1}\right]^{T}$, and the scalar variable $\bar{v}_{i}=v_{i}+\eta_{i}$, where input sequence $\left\{x_{i}\right\}$ is colored 
with variance $\sigma_{x}^{2}$ and $v_{i}$ denotes thewhite Gaussian measurementnoisewith $N\left(0, \sigma_{v}^{2}\right)$, and $\eta_{i}$ is the impulsive measurement noise.The subscript ()$^{T}$ denotes the vector transpose operation. Let $\hat{\mathbf{w}}_{i}$ be an estimate $\mathbf{W}$ at the $i$-th iteration and $e_{i}$ beanapriorimeasurement error, defined as

$$
e_{i}=d_{i}-\mathbf{u}_{i}^{T} \hat{\mathbf{w}}_{i}
$$

In [15], Hampel introduced the concept of the tanh cost function for M-estimator in robust estimation. Using the concept, Wang proposed a robust cost function using the square value of the output error for robust estimation of Principal Components Analysis (PCA) [16]. Similar to this function, we shallsuggest a new cost function using the square value of thenormalized instant output error with respect to the input vectoras follows:

$$
\begin{aligned}
J_{1}\left(\hat{\mathbf{w}}_{i}\right) & =\tanh \left(\left(e_{i} /\left\|\mathbf{u}_{i}\right\|\right)^{2}\right) \\
= & \frac{1-\exp \left(-2\left(e_{i} /\left\|\mathbf{u}_{i}\right\|\right)^{2}\right)}{1+\exp \left(-2\left(e_{i} /\left\|\mathbf{u}_{i}\right\|\right)^{2}\right)}
\end{aligned}
$$

where $\beta>0$ is the slope. $J_{1}\left(\hat{\mathbf{w}}_{i}\right)$ is even, non-negative, and differentiable all $e_{i} /\left\|\mathbf{u}_{i}\right\|$; thus it can be a cost function. When $e_{i} /\left\|\mathbf{u}_{i}\right\|$ is small, Taylor expansions provided the approximation as follows:

$$
J_{1}\left(\hat{\mathbf{w}}_{i}\right)=\left(e_{i} /\left\|\mathbf{u}_{i}\right\|\right)^{2}
$$

From which this function works like as L2 square-mean and the associated algorithm performs like an NLMS algorithm.

If impulsive measurement noise appears, then $e_{i} /\left\|\mathbf{u}_{i}\right\|$ becomes very large, which sends the cost function into 1 , andfurthermore its derivative into zero, which plays a crucial role in achieving robustness over impulsive measurement noise.

\section{B. Proposed Robust NLMS Algorithm}

Using Huber's cost function [17],Vega proposed a robust cost function as follows [11]:

$$
\rho\left(e_{i} /\left\|\mathbf{u}_{i}\right\|\right)= \begin{cases}\frac{1}{2}\left(\frac{e_{i}}{\left\|\mathbf{u}_{i}\right\|}\right)^{2}, & \text { if } \frac{\left|e_{i}\right|}{\left\|\mathbf{u}_{i}\right\|} \leq \sqrt{\delta} \\ \sqrt{\delta_{i}} \frac{\left|e_{i}\right|}{\left\|\mathbf{u}_{i}\right\|}-\frac{\delta_{i}}{2}, & \text { if } \frac{\left|e_{i}\right|}{\left\|\mathbf{u}_{i}\right\|}>\sqrt{\delta} .\end{cases}
$$

This cost function is related to the framework of the constraint of the energy of the filter update at each iteration as

$$
\left\|\hat{\mathbf{w}}_{i+1}-\hat{\mathbf{w}}_{i}\right\|^{2} \leq \delta_{i}
$$

where $\left\{\delta_{i}\right\}$ is a positive sequence. Using the constraint, Vega proposed a robust variable step-size NLMS (RVSS NLMS) algorithm as follows [11]:

$$
\begin{aligned}
& \hat{\mathbf{w}}_{i+1}=\hat{\mathbf{w}}_{i}+\min \left[\frac{\left|e_{i}\right|}{\left\|\mathbf{u}_{i}\right\|}, \sqrt{\delta_{i}}\right] \operatorname{sgn}\left(e_{i}\right) \frac{\mathbf{u}_{i}}{\left\|\mathbf{u}_{i}\right\|}, \\
& \delta_{i+1}=\alpha \delta_{i}+(1-\alpha) \min \left[\frac{e_{i}^{2}}{\left\|\mathbf{u}_{i}\right\|^{2}}, \delta_{i}\right] .
\end{aligned}
$$

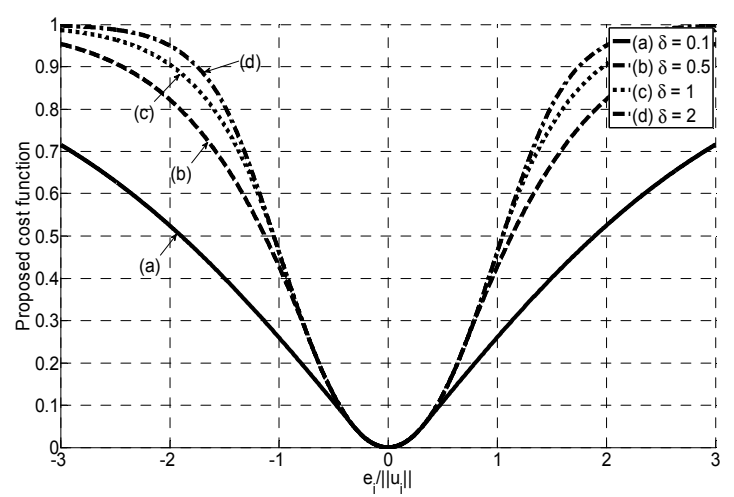

Fig. 1.The proposed cost function $J\left(\hat{\mathbf{w}}_{i}\right)$.

To develop a robust gradient-based adaptive algorithm, which performs better than RVSS NLMS in impulsive-noise Environment, we propose a robust cost function through a combination of the cost functions $J_{1}\left(\hat{\mathbf{w}}_{i}\right)$ and $\rho\left(e_{i} /\left\|\mathbf{u}_{i}\right\|\right)$ as follows:

$$
\begin{aligned}
J\left(\hat{\mathbf{w}}_{i}\right) & =\tanh \left(\rho\left(e_{i} /\left\|\mathbf{u}_{i}\right\|\right)\right) \\
& =\frac{1-\exp \left(-2 \rho\left(e_{i} /\left\|\mathbf{u}_{i}\right\|\right)\right)}{1+\exp \left(-2 \rho\left(e_{i} /\left\|\mathbf{u}_{i}\right\|\right)\right)} .
\end{aligned}
$$

This function also satisfies the conditions of the cost function and like $J_{1}\left(\hat{\mathbf{w}}_{i}\right)$. Let us develop the adaptive algorithm base on the proposed cost function $J\left(\hat{\mathbf{w}}_{i}\right)$ :

$$
\hat{\mathbf{w}}_{i+1}=\hat{\mathbf{w}}_{i}-\nabla_{\hat{\mathbf{w}}_{i}} J\left(\hat{\mathbf{w}}_{i}\right),
$$

where $\nabla_{\hat{\mathbf{w}}_{i}} J\left(\hat{\mathbf{w}}_{i}\right)$ denotes the gradient of $J\left(\hat{\mathbf{w}}_{i}\right)$ with respect to $\hat{\mathbf{w}}_{i}$ as follows:

$$
\nabla_{\hat{\mathbf{w}}_{i}} J\left(\hat{\mathbf{w}}_{i}\right)=\left\{\begin{array}{cc}
-S_{1} \frac{\mathbf{u}_{i}}{\left\|\mathbf{u}_{i}\right\|^{2}} e_{i}, & \text { if } \frac{\left|e_{i}\right|}{\left\|\mathbf{u}_{i}\right\|} \leq \sqrt{\delta_{i}} \\
-S_{2} \frac{\mathbf{u}_{i}}{\left\|\mathbf{u}_{i}\right\|} \operatorname{sgn}\left(e_{i}\right), & \text { if } \frac{\left|e_{i}\right|}{\left\|\mathbf{u}_{i}\right\|}>\sqrt{\delta_{i}}
\end{array},\right.
$$

where

The $\operatorname{sgn}(\bullet)$ denotes the sign function.Fig. 1 shows the proposed cost function $J\left(\hat{\mathbf{w}}_{i}\right)$ for various $\delta$ 's. 


$$
\begin{aligned}
& s_{1} \triangleq \frac{4 \exp \left(-\left(e_{i} /\left\|\mathbf{u}_{i}\right\|\right)^{2}\right)}{\left(1+\exp \left(-\left(e_{i} /\left\|\mathbf{u}_{i}\right\|\right)^{2}\right)\right)^{2}}, \\
& s_{2} \triangleq \frac{4 \exp \left(-2\left(\sqrt{\delta_{i}} \frac{\left|e_{i}\right|}{\left\|\mathbf{u}_{i}\right\|}-\frac{\delta_{i}}{2}\right)\right)}{\left(1+\exp \left(-2\left(\sqrt{\delta_{i}} \frac{\left|e_{i}\right|}{\left\|\mathbf{u}_{i}\right\|}-\frac{\delta_{i}}{2}\right)\right)\right)^{2}} .
\end{aligned}
$$

The proposed algorithm has two-type: an NLMS with the step size $\mu=s_{1}$ or the NLMAD with the step size $\mu=s_{2}$. However, we can insist that the proposed algorithm is VSS NLMS algorithm with step size

$$
\mu_{i}=\left\{\begin{array}{c}
\frac{4 \exp \left(-\left(e_{i} /\left\|\mathbf{u}_{i}\right\|\right)^{2}\right)}{\left(1+\exp \left(-\left(e_{i} /\left\|\mathbf{u}_{i}\right\|\right)^{2}\right)\right)^{2}}, \\
\frac{4 \exp \left(-2\left(\sqrt{\delta_{i}} \frac{\left|e_{i}\right|}{\left\|\mathbf{u}_{i}\right\|}-\frac{\delta_{i}}{2}\right)\right)}{\left(1+\exp \left(-2\left(\sqrt{\delta_{i}} \frac{\left|e_{i}\right|}{\left\|\mathbf{u}_{i}\right\|}-\frac{\delta_{i}}{2}\right)\right)^{2}\right.} \frac{\left\|\mathbf{u}_{i}\right\|}{\left|e_{i}\right|}, \quad \text { if } \frac{\left|e_{i}\right|}{\left\|\mathbf{u}_{i}\right\|}>\sqrt{\delta_{i}}
\end{array}\right.
$$

\section{SimUlation}

In the simulation of channel identification, the order of the adaptive filter is 32 , which is equal to that of the corresponding unknown channel, and $\mathbf{W}$ is randomly generated as a unit vector. The input sequence is an AR1 with pole in 0.85 andsimulation results are obtained from 100 independent trials. The impulsive measurement noise $\eta_{i}$ is added to $y_{i}=\mathbf{u}_{i}^{T} \mathbf{w}$ with $30 \mathrm{~dB}$ signal-to-noise ratio (SNR) and the SNR is calculated by

$$
\mathrm{SNR}=10 \log _{10}\left(\frac{E\left(y_{i}^{2}\right)}{E\left(v_{i}^{2}\right)}\right)
$$

The parameter $\alpha$, the update equation of $\delta_{i}$, and $\eta_{i}$ are assigned as in [11]. $\eta_{i}$ is generated as $\eta_{i}=\omega_{i} N_{i}$, where $\omega_{i}$ is Bernoulli process with $\operatorname{Pr}\left(\omega_{i}=1\right)=p$ and $N_{i}$ is zero-mean Gaussian with power. $\sigma_{N}^{2}=1000 \sigma_{y}^{2}$. For comparison, we use the NLMAD [5] and the robust VSS (RVSS) NLMS [11] algorithm.

First, we study when impulsive measurement noise does not appear. In Fig. 2, the performance of the proposed algorithm is similar tothat of the RVSS NLMS algorithm. Next, we consider the impulsive measurement noise under 2 cases: $p=0.1$ and 0.5 . In Fig. 3 , the proposed algorithm leads to better than those of other compared robust adaptive algorithms.Moreover, Fig. 4 shows that the proposed algorithm leads to the robustness againstimpulsive noise of high probability in robust adaptive algorithms, although the probability is very inadequatecondition in the signal processing applications.

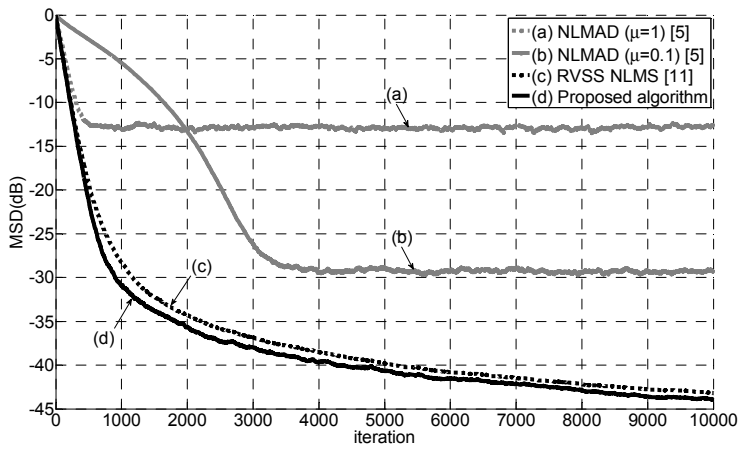

Fig. 2. MSD learning curves (no impulsive measurement noise, $\mathrm{p}=0$ ).

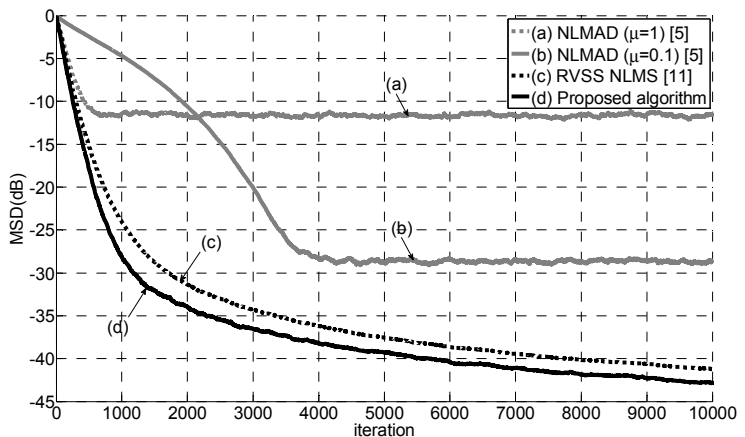

Fig. 3. MSD learning curves with impulsive measurement noise $(\mathrm{p}=0.1)$.

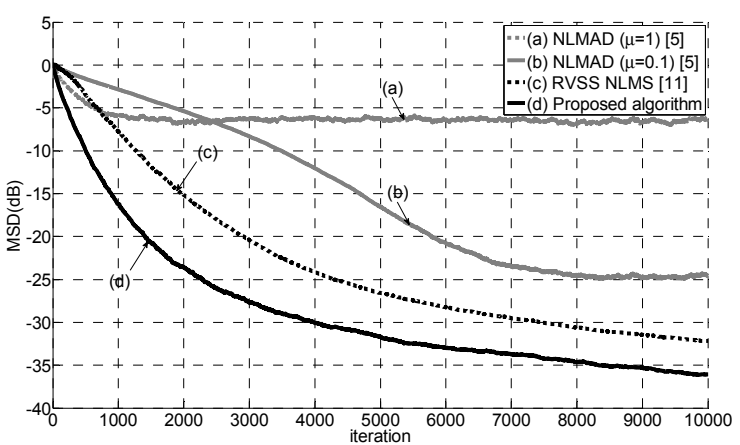

Fig. 3. MSD learning curves with impulsive measurement noise $(\mathrm{p}=0.5)$.

\section{CONCLUSION}

This letter introduces a new gradient-based adaptive filtering algorithm based on a cost function that is constructed by combining two robust cost functions, which are a new tanh-type cost function and Vega's cost function. Through the approach to combine robust cost functions, the robustness of the proposed algorithm outperforms that of other adaptive algorithms. Since the proposed algorithm is derived by combining two robust cost functions, it leads to an excellent transient and steady-state behavior in high probability of impulsive measurement noise. Simulations have shown the performance of the proposed algorithm is better than those of the other robust adaptive algorithms in various probability of impulsive measurement noise.

\section{REFERENCES}

[1] B. Widrow and S. D. Stearns, Adaptive Signal Processing, EnglewoodCliffs, NJ: Prentice-Hall, 1985.

[2] S. Haykin, Adaptive Signal Processing, 4th ed. Upper Saddle River, NJ: Prentice Hall, 2002.

[3] J. Benesty and Y. Huang, Adaptive Signal Processing-Applications toReal World Problems, Berlin, Germany: Springer, 2003. 
[4] Y. Huang, J. Benesty, and J. Chen, Acoustic MIMO Signal Processing, Boston, MA: Springer, 2006.

[5] O. Arikan, A. E. Cetin, and E. Erzin, "Adaptive filtering for non-Gaussian stable processes," IEEE Signal Process. Lett., vol. 1, no. 11,pp. 163-165, Nov. 1994.

[6] C. Kwong, "Dual sign algorithm for adaptive filtering," IEEE Trans.Commun., vol. 34, no. 12, pp. 1272-1275, Dec. 1986.

[7] T. Shao and Y. R. Zheng, "A new variable step-size fractional lowerorder moment algorithm for non-Gaussian interference environments," in Proc. IEEE ISCAS, May 2009, pp. 2065-2068.

[8] Y. R. Zheng and T. Shao, "A variable step-size LMP algorithm for heavy-tailedinterference suppression in phased array radar," in Proc. IEEEAerospace Conf., Mar. 2009, pp. 1-6.

[9] T. Shao, Y. R. Zheng, and J. Benesty, "An Affine Projection Sign AlgorithmRobust Against Impulsive Interferences," IEEE Signal Process.Lett., vol. 17, no. 4, pp. 327-330, Apr. 2010.

[10] J. Chambers and A. Avlonitis, "A robust mixed-norm adaptive filteralgorithm," IEEE Signal Process. Lett., vol. 4, no. 2, pp. 46-48, Feb.1997.

[11] L. R. Vega, H. Rey, J. Benesty, and S. Tressens, "A new robust variablestep-size NLMS algorithm," IEEE Trans. Signal Process., vol. 56, no.5, pp. 1878-1893, May 2008.

[12] P. Petrus, "Robust Huber adaptive filter," IEEE Trans. Signal Process.,vol. 47, no. 4, pp. 1129-1133, Apr. 1999.

[13] Y. Zou, S. C. Chan, and T. S. Ng, "A recursive least M-estimate (RLM)adaptive filter for robust filtering in impulse noise," IEEE Signal Process.Lett., vol. 7, no. 11, pp. 324-326, Nov. 2000.

[14] Y. Zou and S. C. Chan, "A robust quasi-Newton adaptive filteringalgorithm for impulse noise suppression," in Proc. IEEE Inter. Symp.Circuits Syst., May 2001, pp. 677-680.

[15] F. R. Hampel, E. M. Ronchetti, P. J. Rousseeuw, and W. A. Stahel, Robust Statistics: The Approach Based on Inuence Functions, New York: Wiley, 1986.

[16] C. Wang, H. C. Wu, and J. C. Principe, "A cost function for robustestimation of PCA," inProc. SPIE, vol. 2760, pp. 120-127, 1996.

[17] P. J. Huber, Robust Statistics, New York: Wiley, 1981.

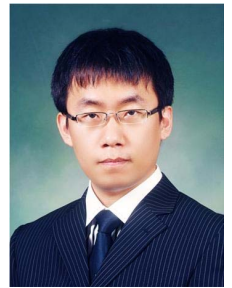

Insun Song received his BS in electrical engineering from Kyungpook national university, Korea, in 2008 and his MS in information technology form Pohang University of Science and Technology (POSTECH), Korea, in 2010 . He is currently studying toward a PhD at POSTECH. His current research interests are image processing and signal processing.

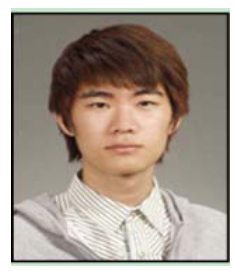

Won Il Lee received his B. S. degree in electronic and electrical engineering from Kyungpook National University in 2010 . He is currently studying toward his Ph.D. at Pohang University of Science and Technology (POSTECH). His current research interests include robust, Linear Parameter Varying (LPV), delayed systems, and Neural Networks.

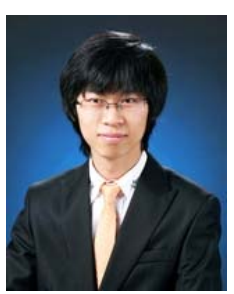

Nam Kyu Kwon received his BS in electrical engineering from Pohang University of Science and Technology (POSTECH) in 2010. He is currently studying toward a $\mathrm{PhD}$ at POSTECH. His current research interests include image processing, robust and Linear Parameter Varying(LPV) control

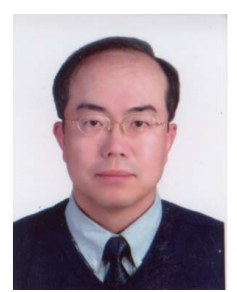

PooGyeon Park received his BS and MSin control and instrumentation engineeringfrom Seoul National University, Korea, in1988 and 1990, respectively, and his $\mathrm{PhDin}$ electrical engineering from Stanford University, in 1995. Since 1996, he has beenaffiliated with the Division of Electrical andComputer Engineering at Pohang Universityof Science and Technology, where he is currentlya professor. His current research interestsinclude robust, Linear Parameter Varying (LPV), Receding Horizon Control (RHC), intelligent, and networkrelatedcontrol theories, signal processing, and wireless communicationsfor a personal area network. 